\title{
T cell immune responses to SARS-CoV-2 and variants of concern (Alpha and Delta) in infected and vaccinated individuals
}

\author{
Stanley C. Jordan ${ }^{1,6}{ }^{凶}$, Bong-Ha Shin ${ }^{1,6}$, Terry-Ann M. Gadsden ${ }^{1}$, Maggie Chu ${ }^{1}$, Anna Petrosyan ${ }^{1}$, Catherine N. Le ${ }^{2}$, Rachel Zabner $^{2}$, \\ Jillian Oft ${ }^{2}$, Isabel Pedraza ${ }^{2}$, Susan Cheng ${ }^{3}$, Ashley Vo ${ }^{1}$, Noriko Ammerman ${ }^{1}$, Jasmine Plummer (iD) ${ }^{4}$, Shili Ge $^{1}$, Max Froch ${ }^{1}$, Anders Berg ${ }^{5}$, \\ Mieko Toyoda ${ }^{1}$ and Ruan Zhang (iD) ${ }^{1}$
}

(c) The Author(s), under exclusive licence to CSI and USTC 2021

Cellular \& Molecular Immunology (2021) 18:2554-2556; https://doi.org/10.1038/s41423-021-00767-9

Our understanding of immune responses to SARS-CoV-2 and variants of concern (VOCs) has been primarily acquired through analysis of Spike-specific lgG responses. However, a more comprehensive understanding of the breadth and longevity of immune responses after infection and vaccination requires analysis of cellular immunity. Herein, we report on T cell immunity in infected and vaccinated individuals, identifying CD4+/CD8+ T cell cytokine responses to SARS-CoV-2 and variant peptides (Alpha, B.1.1.7 and Delta, B.1.617.2). Our results demonstrate that $T$ cells in infected or vaccinated individuals can elicit robust and cross-reactive immune responses against VOCs. This information could be helpful in understanding the composition and durability of human immunity to SARS-CoV-2 and VOCs.

The limited efficacy of most treatments and high death rates associated with SARS-CoV-2 infection have led to a focus on vaccines as the last best hope for stemming the pandemic. However, the persistence of SARS-CoV-2, driven primarily by VOCs, has raised concerns regarding our ability to induce durable immunity through vaccination [1].

Early observations have raised concerns regarding the rapid and unpredictable dissipation of IgG responses to SARS-CoV-2 peptides and the potential effect on long-term immunity $[2,3]$. In addition, reports have shown that IgG spike protein-specific immune responses induced by the BNT162b2 (Pfizer) vaccine may have reduced activity against the Alpha variant [4]. The recent emergence of the Delta variant has reinvigorated concerns about the protective immunity provided by current SARS-CoV-2 vaccines since disturbing reports of vaccine breakthrough infections have been published [5].

Spike-specific IgG receptor-binding domain (RBD) antibodies are evanescent and do not reflect important memory components. Thus, additional analysis of CD4+/CD8 $+T$ cell responses to SARS-CoV-2 spike peptides and VOCs [6] could broaden our understanding of SARS-CoV-2-specific T cell immunity.

The emerging Delta variant is characterized by multiple mutations in the spike protein including T19R, $157-158, L 452 R$, T478K, D614G, P681R, and D950N. It is likely that these mutations affect immune responses to important antigenic regions of the receptor-binding domain. In addition, strains with the P681R mutation have accelerated replication, increasing infectivity. Data on the effectiveness of SARS-CoV-2vaccines against the Delta variant are limited [7].

We developed a whole-blood assay to detect SARS-CoV-2specific $T$ cells. Analysis of cytokine production in stimulated T cells confirmed that IL-2 and TNF- $a$ are consistent markers for activated $\mathrm{CD} 4+\mathrm{T}$ cells, while activated $\mathrm{CD} 8+\mathrm{T}$ cells mainly produce TNF- $a$ and IFN- $\gamma$. After incubating whole blood with a SARS-CoV-2 Spike peptide pool, we were able to identify Spikereactive T cells by dual-cytokine gating (Supplementary Fig. S1). In this assay, healthy individuals with no history of SARS-CoV-2 infection demonstrated no significant $T$ cell response to the SARSCoV-2 spike peptide. However, T cells from SARS-CoV-2-infected or vaccinated individuals showed substantial spike-specific CD4+ and $\mathrm{CD} 8+\mathrm{T}$ cell populations.

Next, we examined memory T cell immunity against SARS-CoV-2 in 134 patients with documented SARS-CoV-2 infection (Supplementary Table S1 and Supplementary Fig. S2). T cell immune responses to peptide pools of 5 major SARS-CoV-2 proteins (Spike, VME, NCAP, AP3A, and NS7A) were analyzed. For healthy control individuals, no significant $C D 4+T$ cell responses to the 5 SARSCoV-2 proteins were seen (Fig. 1A, IL-2+/TNF-a+ (\%) in CD4+ $<0.05 \%$, mean $=0.01 \%$ ). However, $20 \%$ of healthy individuals showed heterogeneous TNF- $a+/$ IFN $-\gamma+$ CD8 + T cell $(>0.05 \%)$ responses to the 5 SARS-CoV-2 proteins, which could represent cross-reactivity among CD8 $+\mathrm{T}$ cells generated during previous endemic coronavirus infection (Fig. 1B) [8]. Based on the background level of the CD4+ T cell response in healthy controls, we set $0.05 \%$ dual-positive CD4+ and CD8+ T cells as the cutoff level for identifying positive T cell immunity against SARS-CoV-2. Overall, we observed that $88 \%$ (30 of 34) of infected patients had either positive CD4+ or CD8+ T cell immunity to one or more of the 5 SARS-CoV-2 proteins. Most patients showed positive CD4+ T cell immunity (85\%, 29 of 34), and CD4+ T cells demonstrated immunodominant responses to Spike peptides, as previously described [8] (Fig. 1A). CD8+ T cells showed similar responses to the 5 proteins.

Next, we analyzed Spike-specific CD4+/CD8+ immune responses induced by the Pfizer BNT162b2 vaccine. We compared Spike-specific

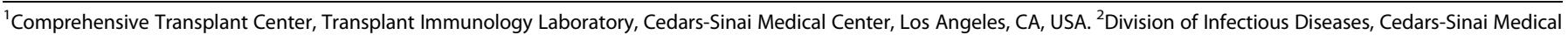

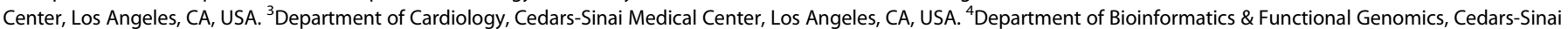

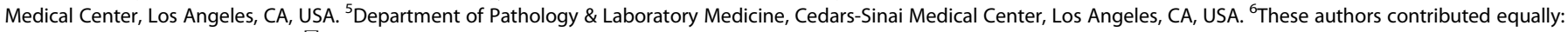
Stanley C. Jordan, Bong-Ha Shin. ${ }^{\bowtie}$ email: stan.jordan@cshs.org

Received: 22 August 2021 Revised: 26 August 2021 Accepted: 27 August 2021

Published online: 16 September 2021 
A
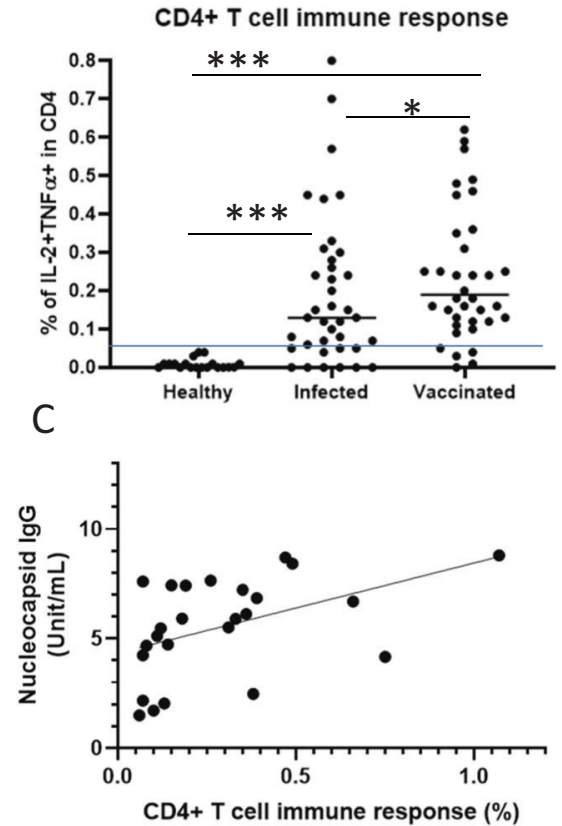

$\mathrm{E}$

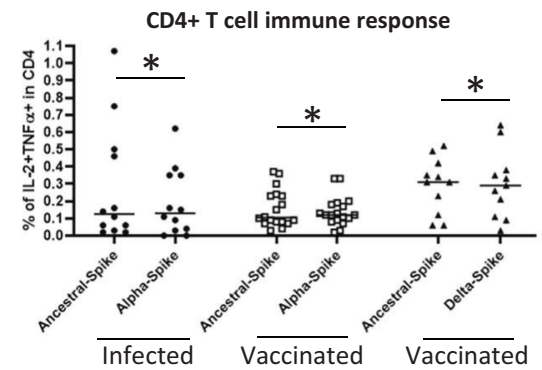

B

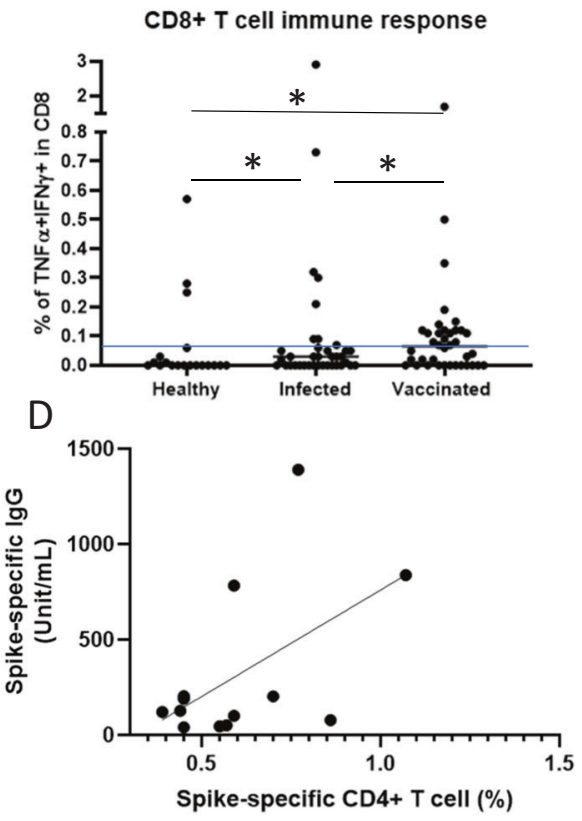

$\mathrm{F}$

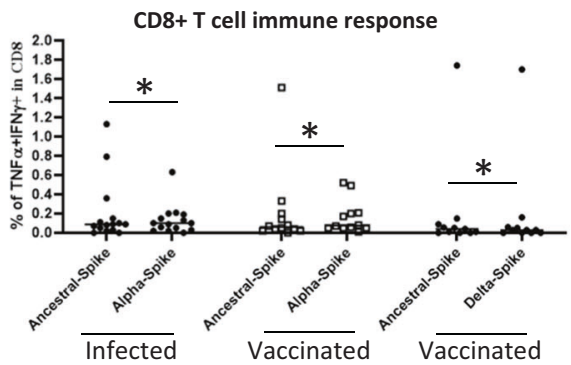

Fig. 1 Immune responses in SARS-CoV-2-infected patients and vaccinated individuals. A, B SARS-CoV-2 Spike-specific CD4+ and CD8+ T cells in healthy, infected and vaccinated individuals. Whole blood was stimulated with Spike peptides, and T cells with dual-cytokine staining were gated. The blue line shows the $0.05 \%$ cutoff. C The correlation of Nucleocapsid-specific lgG titers with CD4+ T cell immune responses to one or more of 5 major SARS-CoV-2 proteins in 25 patients. D The correlation between Spike-specific CD4+ T cell immune responses and Spike-specific Ig levels in 13 patients with elevated CD4+ Spike-specific T cell immune responses (IL-2+/TNF- $\alpha+$ cell\% in CD4+ >0.3\%). E, F T cell immune responses to ancestral and variant spike peptides are shown. CD4+/CD8+ Spike-specific T cells from infected and vaccinated healthy individuals were assessed for reactivity to ancestral, Alpha variant and Delta variant Spike peptides. Each dot represents one individual. ${ }^{*} P=\mathrm{NS} ;{ }^{* * *} p<0.001$.

T cell immunity among 19 healthy controls, 38 infected patients, and 38 vaccinated individuals 1 month after the 2 nd vaccine dose (Fig. $1 \mathrm{~A}$, B). No healthy unvaccinated individuals showed positive CD4 + T cell immunity against SARS-CoV-2, but infected patients and vaccinated individuals demonstrated substantial spike-specific CD4+ T cell immunity, with rates of $87 \%$ (33 of 38 ) and $89 \%$ (34 of 38), respectively. CD8+ T cells from healthy controls, infected patients, or vaccinated individuals showed $21 \%$ (4 of 19), $34 \%$ (13 of 38), and $58 \%$ (22 of 38) positivity for immune responses against SARS-CoV-2 spike peptides, respectively. Therefore, the Pfizer BNT162b2 vaccine induced T cell reactivity to Spike-specific peptides that was equivalent to that seen in infected patients after recovery.

Serum from SARS-CoV-2 patients was submitted for clinical nucleoprotein IgG titering. In 25 patients with nucleocapsid IgG positivity, we analyzed the association with CD4+ T cell immunity to the 5 SARS-CoV-2 peptides (the highest response was compared). Although T cell immunity was not nucleocapsid-specific, there was a significant association between CD4 $+\mathrm{T}$ cell immunity and nucleocapsid lgG titers ( $p=0.022 ; R=0.457$; Fig. 1 C).

We then examined Spike-specific IgG levels in 80 SARS-CoV-2infected patients and compared them to Spike-specific $T$ cell immunity results. In total, $72.58 \%$ of patients with detectable Spike-specific CD4+ T cells were positive for Spike-specific lgG. Among patients without detectable Spike-specific T cell immunity, $61.11 \%$ also were negative for Spike-specific IgG by serology. In 13 patients with high Spike-specific CD4+ T cell immunity (IL-2+/TNF-a+ (\%) in CD4+ $\geq 0.3 \%)$, we also observed a strong correlation between $T$ cell immunity and the level of Spike-specific IgG ( $p=0.0316 ; R=0.5288$; Fig. 1D).

The Alpha (B.1.1.7) variant contains the E484K mutation, which establishes resistance to serologic responses in infected individuals [4]. To determine whether this VOC evades T cell immunity, we analyzed 19 infected/recovered patients and 18 healthy vaccinated individuals for CD4+/CD8 + T cell responses against the Alpha variant Spike protein. As shown in Fig. $1 E, F$, there were no significant reductions in CD4+/CD8 + T cell responses to the Alpha variant Spike peptides compared to those to the ancestral Spike peptides (mean of infected patients: $0.23 \%$ ancestral vs. $0.18 \%$ Alpha variant; mean of vaccinated individuals: $0.16 \%$ ancestral vs. $0.14 \%$ Alpha variant). In addition, nearly identical CD4+ and CD8 $+\mathrm{T}$ cell responses to the Delta variant peptides and SARS-CoV-2 spike peptides were detected in 11 healthy BNT162b2-vaccinated individuals (Fig. 1E, F). In 
summary, T cell memory induced by SARS-CoV-2 infection or vaccination produces similar immune responses against the Alpha and Delta variants. This suggests protective immunity against Alpha and Delta variant infection and possibly infection by other VOCs [9].

Herein, we present data identifying memory $T$ cells with specificity and accuracy for the detection of CD4+/CD8 $+\mathrm{T}$ cell responses to SARS-CoV-2 peptides that differentiate infected and vaccinated individuals from those not exposed to SARS-CoV-2. In addition, analysis of $T$ cell responses to VOCs (Alpha and Delta) showed that SARS-CoV-2 infection and vaccination with BNT162b2 elicited equivalent $\mathrm{T}$ cell responses.

The development of dormancy in memory T cells, B cells, and plasma cells is a natural temporal evolution after infection and/or vaccination that produces populations that can rapidly be activated upon re-exposure to SARS-CoV-2 and are likely to have an important role in preventing or modifying infection by SARSCoV-2 VOCs $[9,10]$. An important consideration in this regard is the dissipation of humoral immunity over time. IgG responses are critical for sterilizing immunity. However, $T$ cell immunity does require an infection to reactivate memory responses. This may result in mild or asymptomatic infections that would be considered "breakthrough" infection. Thus, the level and robustness of $T$ cell memory responses would likely affect the clinical manifestations of the disease.

\section{DATA AVAILABILITY}

Request for data relevant to the manuscript will be considered after assessment and review.

\section{REFERENCES}

1. Krause PR, Fleming TR, Longini IM, Peto R, Briand S, Heymann DL, et al. SARS-CoV2 variants and vaccines. N. Engl J Med. 2021;385:179-86. https://doi.org/10.1056/ NEJMsr2105280.

2. Anand SP, Prévost J, Nayrac $M$, Beaudoin-Bussières $G$, Benlarbi $M$, Gasser $R$, et al. Longitudinal analysis of humoral immunity against SARS-CoV-2 Spike in convalescent individuals up to 8 months post-symptom onset. Cell Rep Med. 2021;2:100290 https://doi.org/10.1016/j.xcrm.2021.100290.

3. Radbruch A, Chang HD. A long-term perspective on immunity to COVID. Nature. 2021. https://doi.org/10.1038/d41586-021-01557-z.

4. Ratcliff J, Nguyen D, Fish M, Rynne J, Jennings A, Williams S, et al. Virological and serological characterization of critically ill patients with COVID-19 in the UK: Interactions of viral load, antibody status and B.1.1.7 variant infection. J Infect Dis. 2021. https://doi.org/10.1093/infdis/jiab283.

5. Brown CM, Vostok J, Johnson H, Burns M, Gharpure R, Sami S. et al. Outbreak of SARS-CoV-2 infections, including COVID-19 vaccine breakthrough infections, associated with large public gatherings - Barnstable County, Massachusetts, July 2021. MMWR Morb Mortal Wkly Rep.2021;70:1059-62. https://doi.org/10.15585/ mmwr.mm7031e2.

6. Xia W, Li M, Wang Y, Kazis LE, Berlo K, Melikechi N, et al. Longitudinal analysis of antibody decay in convalescent COVID-19 patients. Sci Rep. 2021;11:16796 https://doi.org/10.1038/s41598-021-96171-4.
7. Lopez Bernal J, Andrews N, Gower C, Gallagher E, Simmons R, Thelwall S, et al. Effectiveness of Covid-19 vaccines against the B.1.617.2 (Delta) variant. N Engl J Med. 2021;385:585-94. https://doi.org/10.1056/NEJMoa2108891.

8. Peng Y, Mentzer AJ, Liu G, Yao X, Yin Z, Dong D, et al. Broad and strong memory CD4+ and CD8 + T cells induced by SARS-CoV-2 in UK convalescent individuals following COVID-19. Nat Immunol. 2020;21:1336-45.

9. Tarke A, Sidney J, Methot N, Yu ED, Zhang Y, Dan JM, et al. Impact of SARS-CoV-2 variants on the total $C D 4+$ and $C D 8+T$ cell reactivity in infected or vaccinated individuals. Cell Rep Med. 2021;2:100355 https://doi.org/10.1016/j. xcrm.2021.100355.

10. Swadling L, Maini M. T-cells in COVID-19 - united in diversity. Nat Immunol. 2020;21:1307-8.

\section{AUTHOR CONTRIBUTIONS}

$\mathrm{SCJ}$ : Involved in the design and execution of the research and primarily responsible for writing and editing the manuscript. B-HS, PhD: Primarily involved in designing the $T$ cell assay and generating data for the manuscript. T-AMG: Involved in the performance of the T cell and antibody assays. MC: Involved in performance of the T cell and antibody assays. AP: Involved in the performance of the antibody assays. CNL: Involved in gathering and analyzing data and writing the manuscript. RZ: Involved in gathering and analyzing data for the manuscript. JO: Involved in gathering and analyzing data for the manuscript. IP: Involved in gathering and analyzing data for the manuscript. SC: Provided technical and editing support for the project. AV: Provided support for developing the protocol and regulatory documents. NA: Provided regulatory support for the development of the project. JP: Provided laboratory support and editing for the project. SG: Helped in assay development and implementation. MF: Aided in the design and performance of assays. AB: Aided in the development and implementation of antibody assays and the interpretation of data. MT: Helped in the development and implementation of the T cell assay. RZ: Primarily involved in the development of the assays and project and contributed to writing and editing the manuscript

\section{FUNDING INFORMATION}

The funding for this study was derived entirely from internal resources at Cedars-Sinai Medical Center, including funding from the Borstenin Family Foundation, Los Angeles.

\section{COMPETING INTERESTS}

The authors declare no competing interests.

\section{ADDITIONAL INFORMATION}

Supplementary information The online version contains supplementary material available at https://doi.org/10.1038/s41423-021-00767-9.

Correspondence and requests for materials should be addressed to Stanley C. Jordan.

Reprints and permission information is available at http://www.nature.com/ reprints 